\title{
Neuromyelitis Optica in Behçet's Disease Successfully Treated with Radiographic MRI Regression: Case Report
}

Basant Elnady ${ }^{1^{*}}$, Tohamy Elkhouly ${ }^{2}$, Mohamed Alshaar ${ }^{3,4}$, Hawazen Alqahtani ${ }^{3}$, Mohamed Algethami $^{3}$ and Abdulmajeed M. Algethami ${ }^{5}$

${ }^{1}$ Faculty of Medicine Rheumatology and Rehabilitation, Benha University, Egypt

${ }^{2}$ Radiology Department, Benha University hospital, Egypt

3 Internal medicine Departments Al Hada Armed Forces Hospitals, KSA

${ }^{4}$ Neurology department, Al Azhar University, Egypt

${ }^{5}$ Rheumatology Department, Taif University, KSA

"Corresponding author: Basant Elnady, Associate professor, Faculty of Medicine Rheumatology and Rehabilitation, Benha University, Cairo, New Cairo, Egypt, Tel: 00966562386623; E-mail: dr_bossy@yahoo.com

Received date: August 17, 2017; Accepted date: August 29, 2017; Published date: August 31, 2017

Copyright: ( 2017 Elnady B, et al. This is an open-access article distributed under the terms of the Creative Commons Attribution License, which permits unrestricted use, distribution, and reproduction in any medium, provided the original author and source are credited.

\begin{abstract}
Behçet's disease (BD) is a multisystem vasculitis of unknown origin that involves the parenchymal central nervous system (neuro-BD) in less than 10 percent of patients affecting both brain and spinal cord. We reported a rare case of 21-year-old male patient with NBD-myelitis in the form of neuromyelitis optica (NMO) which treated successfully with cyclophosphamide, with marvelous MRI radiographic regression as no disease activity was detected and the patient reported no adverse event, after six cyclophosphamide cycles, cervical MRI marked regression. This case may be an example for decision making and management in such rare cases.
\end{abstract}

Keywords: Behçet's disease; Neuromyelitis optica; MRI; Cyclophosphamide

\section{Introduction}

Neuro-Behçet's disease one of the serious manifestations of Behçet's disease, and spinal cord involvement may appear a rare CNS involvement [1-3]. MRI is helpful to reveal mass-like lesions, isolated brain stem and basal ganglia lesions or spinal cord lesions [4].

$\mathrm{NMO}$, is an idiopathic inflammatory central nervous system demyelinating disease, which can be association with some autoimmune diseases [5]. We report the a rare case of NMO treated with six cycles of cyclophosphamide, with marvelous MRI radiographic regression and hope this case may be an example for decision making and management in such cases.

\section{Case Report}

A 21-year-old male patient wheelchair bound, labeled as juvenile idiopathic arthritis during childhood, and he was off steroid and methotrexate, presented to rheumatology clinic with history of recurrent arthritis (hands and knee), oro-genital ulcers, progressive lower limbs weakness and inability to walk for more than 6 months and diplopia.

Systemic examinations were unremarkable except for oral ulcers, active genital ulcerations and scars of old ulcerations, bilateral pale optic discs on ophthamoscope. Skin pathergy test (SPT) was positive [6].

Neurological examination revealed, bilateral cerebellar pyramidal syndrome sparing the face, affecting lower limbs more than upper limbs, sensory level at T5-6 segment with dissociation, sphincter disturbance.
In the laboratory investigations, complete blood count, renal and liver function tests, electrolytes, serum creatine kinase, creatine kinasemyocardial bound, thyroid hormones, vitamin B12, folic acid, serum immunoglobulin, and complement levels were all normal. Serologic studies for hepatitis $\mathrm{B}$ and $\mathrm{C}$ were all negative.

Rheumatoid factor was positive, but ANA, anti Sm, anti SSA/SSB, anti Scl-70, anti Jo-1, anti U1 RNP, and anti-centromere were all negative. HLA B51 was positive however aquaporin-4 antibody is not available in our institution. CSF was normal and negative for oligoclonal bands.

MRI Brain and lumber-spine were unremarkable however; MRI orbit showed optic neuritis and cervical spines showed long segment of cervical demyelinating lesions (Figure 1) with optic neuritis, clinical and radiological findings go with a diagnosis of Behcet's Disease (BD) with NMO.

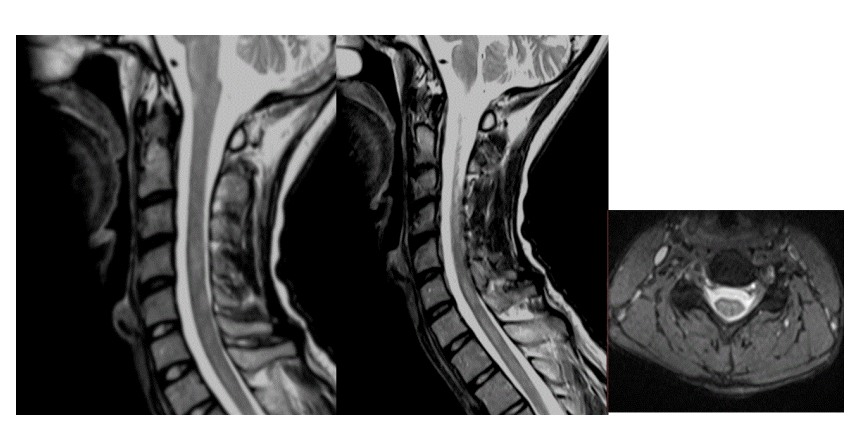

Figure 1: T2W sagittal and axial cervical MRI images, showed long segment of abnormal intramedullary bright $\mathrm{T} 2$ signal. 
Patient treated with pulse steroid (1000 mg methylprednisolone IVI per day) for three days and received cyclophosphamide cycles (1000 $\mathrm{mg} / \mathrm{mg} / \mathrm{m}^{2} / \mathrm{month}$ ) for 6 months and he has improved progressively regarding the weakness, with marvelous MRI regression of the previous findings (Figure 2).

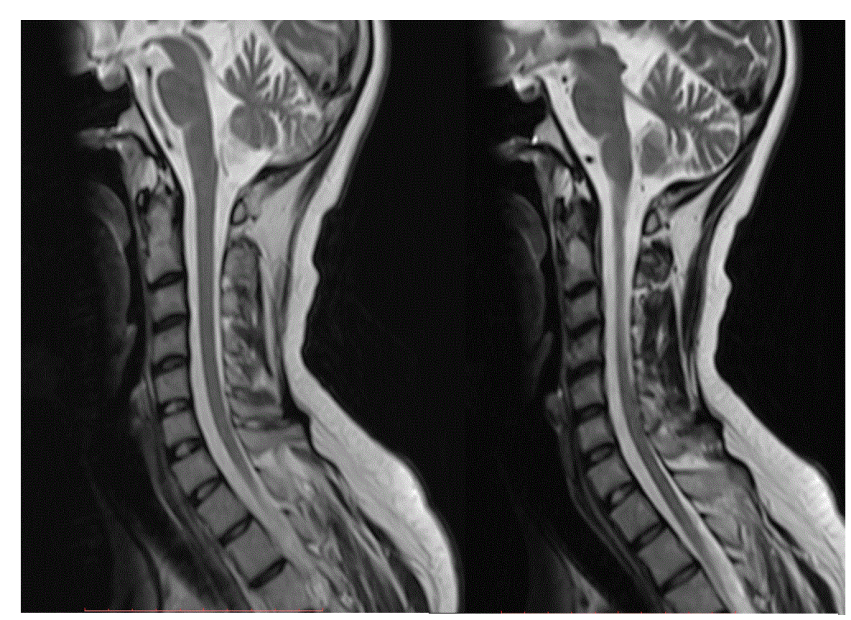

Figure 2: Follow up T2W sagittal cervical MRI images, revealed marked resolution of the previously reported imtramedullary lesions.

\section{Discussion}

Neuro-Behçet disease (NBD) must be considered in the differential diagnosis of stroke in young adults, multiple sclerosis, movement disorders, intracranial hypertension, intracranial Sino venous occlusive diseases, and other neurologic syndromes.

NBD occurs in less than 10 percent of patients with Behçet's syndrome in most series [7-9]. It is observed more frequently in men than women. Both the central and peripheral nervous systems can be involved. Central nervous system manifestations can be divided into 2 main groups: (1) Parenchymal disease is subdivided into brainstem disease, multifocal (diffuse) disease (including brainstem, cerebral, or spinal cord disease), myelopathy, cerebral disease (including encephalopathy, hemiparesis, hemi sensory loss, seizures, dysphagia, and mental changes such as psychosis and cognitive dysfunction), and optic neuropathy. (2) Non-parenchymal disease includes cerebral venous thrombosis, intracranial hypertension syndrome (pseudo tumor cerebri), acute meningeal syndrome, and uncommonly stroke due to arterial thrombosis, dissection, or aneurysm. Peripheral neuropathy and myopathy are relatively rare [10].

Neuromyelitis optica (NMO) is an idiopathic inflammatory central nervous system demyelinating disease, which mainly affects the spinal cord and optic nerves, previously thought to be a variant of multiple sclerosis (MS). Its clinical evolution and histopathological findings showed differently from MS with common association with some other autoimmune diseases [11].

Autoimmune diseases could be the cause of NMO in some patients, other hypothesis consider it may be co-associated as a result of common autoimmune mechanisms or, others may consider as coassociated in a nonspecific way as optic neuritis and myelitis, can occur in some disease as paraneoplastic diseases and systemic autoimmune diseases $[12,13]$.

Over the last two decades, some reported cases of isolated myelitis, especially a longitudinal segment of transverse myelitis and optic neuritis suggestive of NMO were associated to Sjogrens syndrome or SLE [14].

Patients with systemic autoimmune disease who develop long segment transverse myelitis, associated with optic neuritis, confirmed with MRI lesion patterns are very likely to have NMO coexisting, regardless of the result of aquaporin- 4 antibody serologic status. The test for aquaporin- 4 autoantibodies could be suppressed by the concomitant use of immunosuppressive medications and steroids in autoimmune patients, increasing the chances of negative serological test for aquaporin-4 autoantibodies [15]. Owing to the lack of data, there is no established treatment protocol for NBD-myelitis. Corticosteroids considered to be effective for the acute phase. Other immunosuppressive medications such as, methotrexate, azathioprine, cyclophosphamide, may be used [16]. Cyclophosphamide may also favorably influence the course of concomitant NMO [17]. Anti TNF therapy as such as infliximab, etanrcept and adalimumab can be used in treatment of BD especially with eye affection as anterior and posterior uveitis however, demyelination could be an associated side effect. Neurologist should avoid therapies that interfere with tumor necrosis factor-alpha function because they can aggravate or induce demyelination in those patients $[18,19]$.

\section{Conclusion}

$\mathrm{NMO}$ and rheumatologic diseases can be coexisting conditions in autoimmune disease, which are clinically expressed in BD. Neurologists and rheumatologists has an important role to recognize such cases, NMO with BD could be the first CNS event, especially longitudinally extensive transverse myelitis should be suspected. The early therapeutic implications of these relationships should be started as early as possible. Prospective studies of patients with co-existing $\mathrm{NMO}$ and rheumatologic diseases are needed to define these relationships for future better diagnostic and therapeutic decisions.

\section{References}

1. Kantarci O, Siva A (2003) Behçet's disease: diagnosis and management. In: Noseworthy J, editor. Neurological therapeutics: principles and practice. Martin Dunitz Publishers, London, UK.

2. Direskeneli H (2001) Behçet's disease: infectious aetiology, new autoantigens, and HLA-B51. Ann Rheum Dis 60: 996-1002.

3. Siva A, Altintas A, Saip S (2004) Behçet's disease and the nervous system. Curr Opin Neurol 17: 347-357.

4. Serdaroglu P (1998) Behçet's disease and the nervous system. J Neurol 245: 197-205.

5. De Seze J, Lebrun C, Stojkovic T, Ferriby D, Chatel M, et al. (2003) Is Devicás neuromyelitis optica a separate disease? A comparative study with multiple sclerosis. Mult Scler 9: 521-525.

6. Study Group for Behçet's Disease (1990) Criteria for diagnosis of Behçet's disease. Lancet 335: 1078-1080.

7. Akman-Demir G, Serdaroglu P, Tasçi B (1999) Clinical patterns of neurological involvement in Behçet's disease: evaluation of 200 patients. The Neuro-Behçet Study Group. Brain 122: 2171-2182.

8. Siva A, Saip S (2009) The 0tial diagnosis. J Neurol 256: 513.

9. Al-Araji A, Kidd DP (2009) Neuro-Behçet's disease: epidemiology, clinical characteristics, and management. Lancet Neurol 8: 192-204. 
Citation: Elnady B, Elkhouly T, Alshaar M, Alqahtani H, Algethami M, et al. (2017) Neuromyelitis Optica in Behçet's Disease Successfully Treated with Radiographic MRI Regression: Case Report. J Arthritis 6: 250. doi:10.4172/2167-7921.1000250

Page 3 of 3

10. Kalra S, Silman A, Akman-Demir G, Bohlega S, Borhani-Haghighi A, et al. (2014) Diagnosis and management of Neuro-Behçet's disease: international consensus recommendations. J Neurol 261: 1662-1676.

11. Cross SA, Salomao DR, Parisi JE, Kryzer TJ, Bradley EA, et al. (2003) Paraneoplastic autoimmune optic neuritis with retinitis defined by CRMP-5IgG. Ann Neurol 54: 38-50.

12. Keegan BM, Pittock SJ, Lennon VA (2008) Autoimmune myelopathy associated with collapsing response-mediator protein-5 immunoglobulin G. Ann Neurol 63: 531-534.

13. Pimentel ML (2014) Neuromyelitis optica spectrum disorder and Sjögren syndrome are overlapping disorders and participate in the same autoimmunity context?. Arq Neuropsiquiatr 72: 577-579.

14. Wingerchuk DM, Weinshenker BG (2012) The emerging relationship between neuromyelitis optica and systemic rheumatologic autoimmune disease. Mult Scler 18: 5-10.

15. Mok CC, To CH, Mak A, Poon WL (2008) Immunoablative cyclophosphamide for refractory lupus-related neuromyelitis optica. J Rheumatol 35: 172-174.
16. Ait Ben Haddou EH, Imounan F, Regragui W, Mouti O, Benchakroune N, et al. (2012) Neurological manifestations of Behcet's disease: evaluation of 40 patients treated by cyclophosphamide. Rev Neurol (Paris) 168: 344-349.

17. Minagar A, Sheremata WA (2000) Treatment of Devic's disease with methotrexate and prednisone. Int J MS Care 2: 39-43.

18. Sfikakis PP, Markomichelakis N, Aplosy E, Assaad-Khalil S, Bodaghi B, et al. (2007) Anti-TNF therapy in the management of Behçet's disease-review and basis for recommendation. Rheumatology (Oxford) 46: 736-741.

19. Caso F, Costa L, Rigante D, Lucherini OM, Caso P, et al. (2014) Biological Treatments in Behçet's Disease: Beyond Anti-TNF Therapy. Mediators Inflamm 2014: 107421. 